\title{
Une comparaison entre des blés hybrides produits par voie chimique et leurs parents : niveaux d'hétérosis et élaboration du rendement
}

\author{
FX Oury *, J Koenig, P Bérard, M Rousset \\ INRA, station d'amélioration des plantes, Domaine de Crouelle, 63000 Clermont-Ferrand, France
}

(Reçu le 31 octobre 1989 ; accepté le 22 février 1990)

\begin{abstract}
Résumé - Le regroupement des résultats de 5 années d'expérimentation a permis la comparaison de 34 hybrides de blé tendre d'hiver avec leurs parents. Ce matériel a été évalué dans des dispositifs en blocs complets randomisés, la parcelle élémentaire étant de $4 \mathrm{~m}^{2}$. Globalement, l'hétérosis se manifeste au niveau du rendement, de la biomasse aérienne, et du poids de 1000 grains. Par ailleurs les $F_{1}$ sont, en moyenne, proches du parent supérieur pour la hauteur, au niveau du parent moyen pour le nombre d'épis par $\mathrm{m}^{2}$, la fertilité de l'épi, et le harvest-index, et proches du parent inférieur pour la précocité. Le rendement de l'hybride est corrélé à l'hétérosis sur le rendement, et n'est pas corrélé au rendement des parents, ce qui peut être lié à l'homogénéité de notre échantillon parental, les lignées étant toutes de bonne valeur propre. A peuplement épis égal, une $F_{1}$ ne produit pas plus de grains par épi qu'une lignée; par contre, à nombre de grains par $\mathrm{m}^{2}$ égal, l'hybride remplit mieux ses grains que la lignée. Le rendement accru des $F_{1}$ est lié à une augmentation de la production de biomasse aérienne : la voie hybride pourrait donc permettre d'améliorer la productivité du blé autrement que par une augmentation du harvest-index.
\end{abstract}

blé hybride / hétérosis / élaboration du rendement

Summary - A comparison between wheat hybrids produced using a chemical hybridizing agent and their parents: levels of heterosis and yield elaboration. The regrouping of data from 5 years of experimentation enabled a comparison to be made of 34 winter wheat hybrids with their parents. This material has been evaluated in randomised complete block designs, the elementary plot surface being $4 \mathrm{~m}^{2}$. Globally, heterosis appears for yield (on average the 34 hybrids yield $3.1 \%$ more grain than their best-parent yield; and the best hybrid yields $7.3 \%$ more than the best line), biomass production, and 1000 seed weight (fig 1, table III). In other respects $F_{1}$ are, on average, close to the high-parent for height (fig 1, table III), on a par with mid-parent for number of ears per $m^{2}$, fertility of ear, and harvest-index (fig 2, table III), and close to the low-parent for earliness (fig 3, table III). Hybrid yield is correlated with heterosis for yield, and not correlated with the yield of parents (table VI). This may be linked with the parental sample homogeneity (when the lines are all of good per se value, it appears that it is difficult to predict the $F_{1}$ yield from the parental yields). With an identical number of ears per $m^{2}$, a $F_{1}$ does not produce more grains per ear than a line (fig 4 , table VII); on the other hand, with the same number of grains per $\mathrm{m}^{2}$, the hybrid fills its grains better than the line (fig 5, table VII). Otherwise, the greater yield of the $F_{1}$ is related to an increase in biomass production (tables VIII, IX): thus hybrid breeding could give improvement in wheat productivity different to that resulting from an increase in harvestindex.

hybrid wheat / heterosis / yield elaboration

\section{INTRODUCTION}

Les difficultés liées à la production de semences $F_{1}$, chez une plante autogame et à faible taux de multiplication, ont, pendant longtemps, limité les possibilités d'expérimentation sur les blés hybrides. Les résultats concernant les niveaux d'hétérosis étaient souvent obtenus dans des dispositifs avec semis en poquets (Briggle et al, 1964; Akemine et Kumagai, 1966; Bitzer et al,
1982), ou semis sur une seule ligne avec un espacement entre plantes très important (Borojevic, 1966; Planchon et Diehl, 1967; Sharma et Ahmad, 1978). Ils étaient donc difficilement extrapolables aux conditions correspondant aux pratiques culturales habituelles. De plus, le nombre d'hybrides testés était parfois très faible (Briggle et al, 1964; Briggle et al, 1967; Planchon et Diehl, 1967; Jost et Hayward, 1980), ce qui interdisait une généralisation des résultats obtenus.

\footnotetext{
* Correspondance et tirés à part
} 
L'apparition d'agents chimiques d'hybridation (produits qui induisent, par pulvérisation sur les plantes à un stade phénologique approprié, une stérilité mâle, Rousset, 1984) a permis, ces 10 dernières années, de produire de nombreuses combinaisons hybrides, en disposant de quantités de semences suffisantes pour des expérimentations en microparcelles, dans des conditions proches de la pratique agricole courante.

De plus, l'obtention des semences $F_{1}$ par voie chimique est rapide, ce qui permet d'utiliser des parents équivalents aux variétés les plus performantes du moment. Ce n'est pas le cas lorsque les hybrides sont produits en utilisant la stérilité mâle génocytoplasmique. En effet, les délais nécessaires pour obtenir les lignées mâle-stériles et les lignées restauratrices sont très longs (Rousset, 1986; Glenn, 1987). En conséquence, au moment de la production de l'hybride les parents ne sont plus du niveau des meilleures variétés lignées pures, car celles-ci ont entretemps bénéficié des travaux de la sélection classique (Edwards, 1987). La production d'hybrides au moyen de gamétocides a donc permis de comparer des hybrides et des lignées à un même niveau de progrès génétique.

Dans cet article, nous rapportons les résultats d'essais visant à comparer des hybrides à leurs parents. Ces expérimentations ont eu lieu en $1978,1980,1981,1982$ et 1985, à la station d'amélioration des plantes de Clermont-Ferrand, et mettaient à chaque fois en œuvre des combinaisons hybrides obtenues, par voie chimique, à partir de lignées du commerce ou du laboratoire choisies parmi les meilleures du moment. Nous avons étudié les manifestations de l'hétérosis pour différents caractères, en particulier le rendement et la production de biomasse aérienne. Par ailleurs, l'étude des composantes du rendement nous a permis une approche de son élaboration chez l'hybride.

\section{MATÉRIEL ET MÉTHODES}

Les expérimentations ont permis la comparaison de 28 combinaisons hybrides différentes avec les 34 parents correspondants (ces combinaisons figurent dans le tableau I). Les lignées Champlein (en 1980), Talent et Arminda (en 1981), ont été observées en plus des parents d'hybrides. L'ensemble des $F_{1}$ a été obtenu par voie chimique (gamétocide Rhom \& Haas), sauf C2-6-11 x Champlein-Trf et C2-6-11 x Maris-Beacon en 1978, qui ont été produits en utilisant la stérilité mâle induite par le cytoplasme de Triticum timopheevi. La pureté des hybrides était très satisfaisante (en moyenne $95 \%$ d'hybrides pour les combinaisons étudiées), sauf dans les cas de $\mathrm{C} 2107 \times$ Beauchamp en 1981, et C8-14-2 x Clément en 1982, pour lesquels le taux de plantes de type femelle ou hors-type atteignait $20 \%$. Cependant, ce taux ne nous a pas paru devoir justifier la non-prise en compte de ces $2 F_{1}$. La plupart des combinaisons hybrides (21 sur 28 ) faisaient intervenir un parent demi-nain.

Les hybrides et leurs parents ont été étudiés dans des dispositifs en blocs complets randomisés ( 2 blocs en 1982,3 en 1980 et 1981,4 en 1985 , et 6 en 1978), la parcelle élémentaire étant constituée de 5 lignes de $3 \mathrm{~m}$, espacées de $0,25 \mathrm{~m}$. Les techniques culturales étaient proches des méthodes intensives pratiquées en Limagne, avec en particulier $\mathrm{N}, \mathrm{P}, \mathrm{K}$ non limitants, et couverture fongicide. La dose de semis de 250 grains $/ \mathrm{m}^{2}$ a permis d'atteindre l'objectif de peuplement qui était de 200 à 250 plantes $/ \mathrm{m}^{2}$.

Les caractères mesurés ont été la matière sèche totale (MST), le rendement en grains à 15\% d'humidité $(R D T)$, le nombre d'épis par $\mathrm{m}^{2}$ (NÉ), le poids de 1000 grains (PMG), le nombre de grains par $\mathrm{m}^{2}$ (NG) (sauf en 1980 et 1985, où les valeurs de ce caractère ont été calculées à partir de RDT et $P M G$ ), la hauteur $(H T)$, et la précocité d'épiaison (PRÉC). Le nombre de grains par épi (NGÉ) a été calculé à partir de $N G$ et $N E$, et le harvest-index (HI) à partir de RDT et MST.

Tableau I. Hybrides étudiés pendant les différentes années d'expérimentations.

Hybrides testés

Année d'expérimentation

\begin{tabular}{ll} 
C1-2-3 x Champlein & 1978 \\
C1-2-3 x Florent & 1978 \\
C2-6-11 x Champlein-Trf & 1978 \\
C2-6-11 x Maris-Beacon & 1978 \\
C6-2-4 x Clément & 1980 \\
C6-2-4 Hardi & 1980 \\
C45-6 x Clément & 1980 \\
C45-6 x Hardi & 1980 \\
C62-2 x Clément & $1980,1981,1982$ \\
C62-2 x Hardi & 1980 \\
C8-14-2 x Clément & $1980,1981,1982,1985$ \\
C8-14-2 x Hardi & 1980 \\
C8-14-2 x Blueboy & 1980 \\
Talent x Roazon & 1980 \\
C484-2 x Clément & 1981 \\
C2107 x Tarasque & 1981 \\
C8-14-2 x Armada & 1981 \\
C62-2 x Armada & 1981 \\
C2107 x Beauchamp & 1981 \\
C10-1-6 x Fidel & 1981 \\
Frandoc x Festin & 1982 \\
C8-14-2 x Arminda & 1982 \\
C62-2 x Arminda & 1982 \\
C2107 x Fidel & 1982 \\
C6-2-4 x Arminda & 1982 \\
Courtot x Fidel & 1982,1985 \\
R3-7 x Arminda & 1985 \\
C8-14-2 x Camp-Rémy & 1985 \\
\hline &
\end{tabular}


Tous ces caractères ont été mesurés sur des prélèvements $\left(0,375 \mathrm{~m}^{2}\right.$ en $1978 ; 2 \times 0,125 \mathrm{~m}^{2}$ en 1980,1981 et 1982), sauf en 1985 où MST et RDT ont été obtenus par récolte des 3 lignes centrales, et $N E ́$ par comptage des épis sur une ligne complète.

L'existence de génotypes communs à différentes années d'expérimentation nous a permis une caractérisation de l'effet «année», le modèle d'analyse de variance utilisé étant :

$$
y_{i j k}=m+a_{i}+b_{i j}+g_{k}+a_{i} \cdot g_{k}+e_{i j k}
$$

avec $m=$ moyenne générale

$$
\begin{aligned}
& a_{i}=\text { effet année } \\
& b_{i j}=\text { effet bloc, hiérarchisé à l'effet année } \\
& g_{k}=\text { effet génotype } \\
& a_{i} g_{k}=\text { interaction année } \times \text { génotype } \\
& e_{i j k}=\text { erreur résiduelle }
\end{aligned}
$$

Les traitements statistiques ont été réalisés à l'aide des logiciels Amance (Bachacou et al, 1981); et Consistent System (en interface avec GPGS pour la partie graphique).

\section{RÉSULTATS ET DISCUSSION}

Les carrés moyens de l'interaction annéegénotype étant très inférieurs aux carrés moyens de l'effet année (tableau II), nous avons pu intro- duire une correction année, égale à l'écart à la moyenne générale, pour les différentes variables. Ce contrôle de l'effet année nous a ainsi permis de traiter de manière synthétique l'ensemble des données pluriannuelles.

\section{Description de l'hétérosis pour les différents caractères étudiés}

Les valeurs mesurées sur les hybrides sont représentées, en même temps que celles obtenues pour leurs parents, sur les figures 1, 2 et 3 .

La figure 1 regroupe les graphes correspondant aux caractères pour lesquels il y a globablement un effet d'hétérosis : la plupart des 34 hybrides sont situés au-dessus du parent moyen (voir également tableau III). En moyenne les $F_{1}$ produisent plus de biomasse, et ont des rendements et des poids de 1000 grains plus élevés, que leur parent supérieur (tableau III). Leur hauteur est en général proche de celle du parent supérieur, ce qui confirme les observations de Ingold (1974), qui a montré que les combinaisons hybrides faisant intervenir un parent demi-nain

\begin{tabular}{|c|c|c|c|c|c|}
\hline & \multicolumn{5}{|c|}{ Carrés moyens } \\
\hline & $\begin{array}{l}\text { Génotype } \\
\qquad \begin{array}{c}4 d d l \\
(2 d d l)\end{array}\end{array}$ & $\begin{array}{l}\text { Année } \\
2 d d l \\
(3 d d)\end{array}$ & $\begin{array}{c}\text { Bloc } \\
5 d d l \\
(8 d d l)\end{array}$ & $\begin{array}{c}\text { Interaction } \\
\text { année } \times \text { génotype } \\
8 \text { ddl } \\
(6 d d)\end{array}$ & $\begin{array}{l}\text { Erreur } \\
20 d d l \\
(16 d d l)\end{array}$ \\
\hline$M S T$ & $\begin{array}{l}3280^{* *} \\
\left(4682^{* *}\right)\end{array}$ & $\begin{array}{l}4805^{* *} \\
\left(3449^{* *}\right)\end{array}$ & $\begin{array}{c}222 \\
(341)\end{array}$ & $\begin{array}{l}201 \\
(509 *)\end{array}$ & $\begin{array}{l}260 \\
(141)\end{array}$ \\
\hline$R D T$ & $\begin{array}{l}181^{*} \\
\left(147^{*}\right)\end{array}$ & $\begin{array}{l}782^{* *} \\
\left(896^{* *}\right)\end{array}$ & $\begin{array}{l}36 \\
(55)\end{array}$ & $\begin{array}{c}55 \\
\left(219^{* *}\right)\end{array}$ & $\begin{array}{l}54 \\
(33)\end{array}$ \\
\hline$N E$ & $\begin{array}{l}8163^{*} \\
(2465)\end{array}$ & $\begin{array}{c}12567^{\star *} \\
\left(38861^{* *}\right)\end{array}$ & $\begin{array}{l}2351 \\
\text { (3 394) }\end{array}$ & $\begin{array}{l}2840 \\
(1668)\end{array}$ & $\begin{array}{l}2132 \\
(1958)\end{array}$ \\
\hline$N G$ & $\begin{array}{l}49886198^{\star \star} \\
\left(20642981^{* \star}\right)\end{array}$ & $\begin{array}{l}65912792^{* *} \\
\left(78539327^{* \star}\right)\end{array}$ & $\begin{array}{c}2697441 \\
\text { (4 } 242087)\end{array}$ & $\begin{array}{c}4158288 \\
(8305889 \text { *) }\end{array}$ & $\begin{array}{c}3674980 \\
(2457753)\end{array}$ \\
\hline$N G E$ & $\begin{array}{l}323^{* *} \\
\left(65^{*}\right)\end{array}$ & $\begin{array}{l}464^{* *} \\
\left(218^{* *}\right)\end{array}$ & $\begin{array}{l}10 \\
(11)\end{array}$ & $\begin{array}{l}30^{* *} \\
(33)\end{array}$ & $\begin{array}{r}8 \\
(13)\end{array}$ \\
\hline$P M G$ & $\begin{array}{l}310^{* *} \\
\left(190^{* *}\right)\end{array}$ & $\begin{array}{l}139^{* *} \\
\left(145^{* \star}\right)\end{array}$ & $\begin{array}{c}1 \\
\text { (1) }\end{array}$ & $\begin{array}{l}2 \\
\left(6^{* *}\right)\end{array}$ & $\begin{array}{c}1 \\
(1)\end{array}$ \\
\hline
\end{tabular}
ne dépassaient généralement pas la taille du parent supérieur. Tous ces résultats sont

Tableau II. Analyses de variance pour les 5 génotypes communs aux expérimentations de 1980, 1981, 1982; entre parenthèses, même chose pour les 3 génotypes communs aux années 1980,1981, 1982 et 1985 . ${ }^{*}=$ significatif au seuil de 5\%; ${ }^{\star \star}$ significatif au seuil de $1 \%$. 

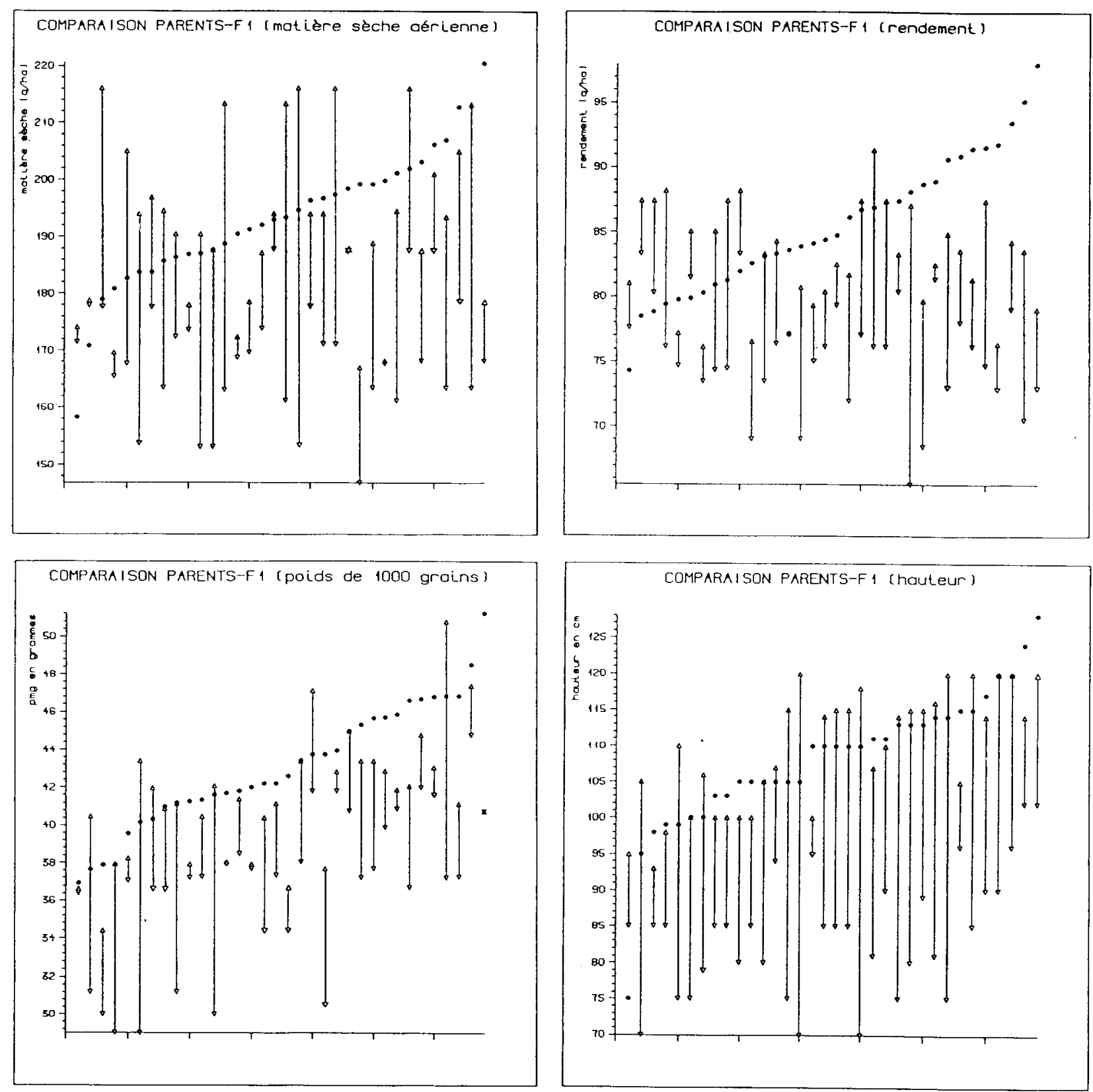

Fig 1. Valeurs observées pour 34 triplets constitués par 1 hybride ("), son parent inférieur $(\nabla)$, et son parent supérieur $(\Delta)$, pour 4 variables (MST, RDT, PMG et HT) présentant globalement un effet d'hétérosis. Les triplets hybride + parents sont disposés selon les valeurs croissantes des $F_{1}$.

conformes à ceux de Goujon et Paquet (1968), Keydel (1973), Borghi et al (1988) et Morgan et al (1989).

On constate (fig1) que pour MST, RDT, PMG et $H T$, c'est sur des hybrides que la valeur maximale du caractère a été enregistrée. Cependant, pour MST, PMG et $H T$, les valeurs de la meilleure lignée et du meilleur hybride sont du même ordre de grandeur, et c'est seulement pour le rendement que plusieurs hybrides dépas- sent nettement la meilleure lignée. C'est ainsi que le rendement du meilleur hybride est supérieur de $7,3 \%$ à celui de la meilleure lignée. Par ailleurs, le rendement moyen des $F_{1}$ est de 85,7 $\mathrm{q} / \mathrm{ha}$, alors que celui des lignées est de 78,6 q/ ha.

La figure 2 regroupe les graphes correspondant aux caractères pour lesquels les hybrides sont répartis de manière à peu près équivalente de part et d'autre du parent moyen, et pour les- 
Tableau III. Hétérosis par rapport au parent moyen et au parent supérieur (et écart type de ces hétérosis), et répartition en effectifs des 34 hybrides $(H)$ par rapport aux parents moyen (PM) et supérieur (PS), pour les 9 caractères étudiés $(M S T=$ matière sèche aérienne totale; $R D T=$ rendement en grains; $H I=$ harvest-index; $N E ́$ = nombre d'épis par $\mathrm{m}^{2} ; N G=$ nombre de grains par $\mathrm{m}^{2} ; N G E$ = nombre de grains par épi; $P M G=$ poids de 1000 grains; $H T=$ hauteur; PRÉC : précocité).

\begin{tabular}{|c|c|c|c|c|c|c|c|c|c|c|}
\hline & \multicolumn{3}{|c|}{$\begin{array}{c}\text { Hétérosis } \\
\text { (\% parent moyen) }\end{array}$} & \multicolumn{3}{|c|}{$\begin{array}{c}\text { Hétérosis } \\
\text { (\% parent supérieur) }\end{array}$} & \multirow[t]{2}{*}{$H>P S$} & \multirow[b]{2}{*}{$P S \geq H>P M$} & \multirow[t]{2}{*}{$P M \geq H \geq P I$} & \multirow[b]{2}{*}{$P l>H$} \\
\hline & $\operatorname{mini}$ & moyen $(\sigma)$ & $\max i$ & $\min i$ & myen $(\sigma)$ & $\operatorname{maxi}$ & & & & \\
\hline$M S T$ & $-9,1$ & $\begin{array}{l}7 \\
(8,3)\end{array}$ & 27,2 & $-17,2$ & $\begin{array}{c}0,7 \\
(9,2)\end{array}$ & 23,5 & 18 & 10 & 4 & 2 \\
\hline$R D T$ & $-8,1$ & $\begin{array}{l}8 \\
(8,9)\end{array}$ & 28,9 & $-10,2$ & $\begin{array}{c}3,1 \\
(8,4)\end{array}$ & 23,9 & 21 & 7 & 1 & 5 \\
\hline$H I$ & $-5,6$ & $\begin{array}{c}0,5 \\
(3,2)\end{array}$ & 7,1 & $-13,6$ & $\begin{array}{r}-5,8 \\
(4,6)\end{array}$ & 1,7 & 6 & 19 & 8 & 1 \\
\hline$N E ́$ & $-14,6$ & $\begin{array}{c}-0,3 \\
(8,8)\end{array}$ & 20,7 & $-24,2$ & $\begin{array}{l}-6,6 \\
(9,1)\end{array}$ & 16 & 5 & 10 & 9 & 10 \\
\hline$N G$ & $-21,8$ & $\begin{array}{c}0,2 \\
(9,2)\end{array}$ & 19,3 & -25 & $\begin{array}{c}-5,4 \\
(9,8)\end{array}$ & 17,5 & 12 & 5 & 7 & 10 \\
\hline$N G E$ & $-18,5$ & $\begin{array}{c}2,8 \\
(11,2)\end{array}$ & 27,7 & $-25,6$ & $\begin{array}{l}-4,6 \\
(11,7)\end{array}$ & 18,9 & 12 & 4 & 12 & 6 \\
\hline$P M G$ & $-1,7$ & $\begin{array}{c}10,4 \\
(6,7)\end{array}$ & 28,3 & $-7,8$ & $\begin{array}{c}4,3 \\
(7,4)\end{array}$ & 25,7 & 26 & 7 & 1 & 0 \\
\hline$H T$ & $-16,7$ & $\begin{array}{l}11,7 \\
(6)\end{array}$ & 19,6 & $-21,1$ & $\begin{array}{r}-1,1 \\
(6,6)\end{array}$ & 10 & 13 & 20 & 0 & 1 \\
\hline PRÉC & $-3,9$ & $\begin{array}{c}-0,8 \\
(1)\end{array}$ & 1,7 & $-3,9$ & $\begin{array}{c}-1,5 \\
(1) \\
\end{array}$ & 0 & 0 & 4 & 20 & 10 \\
\hline
\end{tabular}

quels il n'y a donc globalement pas d'hétérosis (voir également tableau III). Les $\mathrm{F}_{1}$ sont proches du parent moyen pour le peuplement-épis, la fertilité de l'épi, et le harvest-index (tab III), ce qui rejoint les constatations déjà faites par Goujon et Paquet (1968), Keydel (1973), ainsi que Morgan et al (1989) (sauf en ce qui concerne la fertilité de l'épi, pour laquelle ces derniers ont observé un hétérosis significatif).

Dans le cas de NG (fig 2), c'est un hybride qui présente la valeur maximale du caractère. Cependant, les meilleures lignées ont des nombres de grains par $\mathrm{m}^{2}$ tout à fait comparables. Par contre pour $N E ́, N G E ́$ et $H I$, ce sont des lignées qui obtiennent les valeurs maximales, et on ne trouve pas d'hybrides dans les zones correspondant aux forts peuplements-épis, aux fortes fertilités de l'épi, et aux harvest-index élevés. Pour ce dernier caractère, ce résultat était prévisible, car aucune combinaison hybride ne faisait intervenir 2 parents ayant les mêmes gènes de nanisme : ces gènes étant récessifs, il n'y avait donc pas d'hybrides demi-nains dans l'échantillon étudié.

La figure 3 présente le cas de la précocité d'épiaison : les $F_{1}$ sont presque toutes plus précoces que le parent moyen (voir également tableau III), ce qui va dans le sens des résultats de Borojevic (1966), Sharma et Ahmad (1978) et Borghi et al (1988).

Le sens des déviations que nous avons observées pour le rendement et ses composantes est, d'une manière générale, similaire à celui rapporté dans la littérature pour des expérimentations du même type que les nôtres, c'està-dire faisant intervenir un nombre relativement important d'hybrides, observés dans des dispositifs parcellaires. Nos résultats soulignent ainsi les grandes tendances. Cependant, l'amplitude de variation de l'hétérosis est importante (tableau III), et des combinaisons hybrides particulières peuvent, par exemple, présenter un hétérosis pour le peuplement-épis et-ou pour la fertilité de l'épi, et pas pour le poids de 1000 grains (Nettevich, 1968; Jost et Hayward, 1980).

\section{Stabilité de l'hybride pour les différentes varlables étudiées}

Trois combinaisons hybrides ont été observées sur plusieurs années. On constate (tableau IV) que les niveaux d'hétérosis sont très variables 

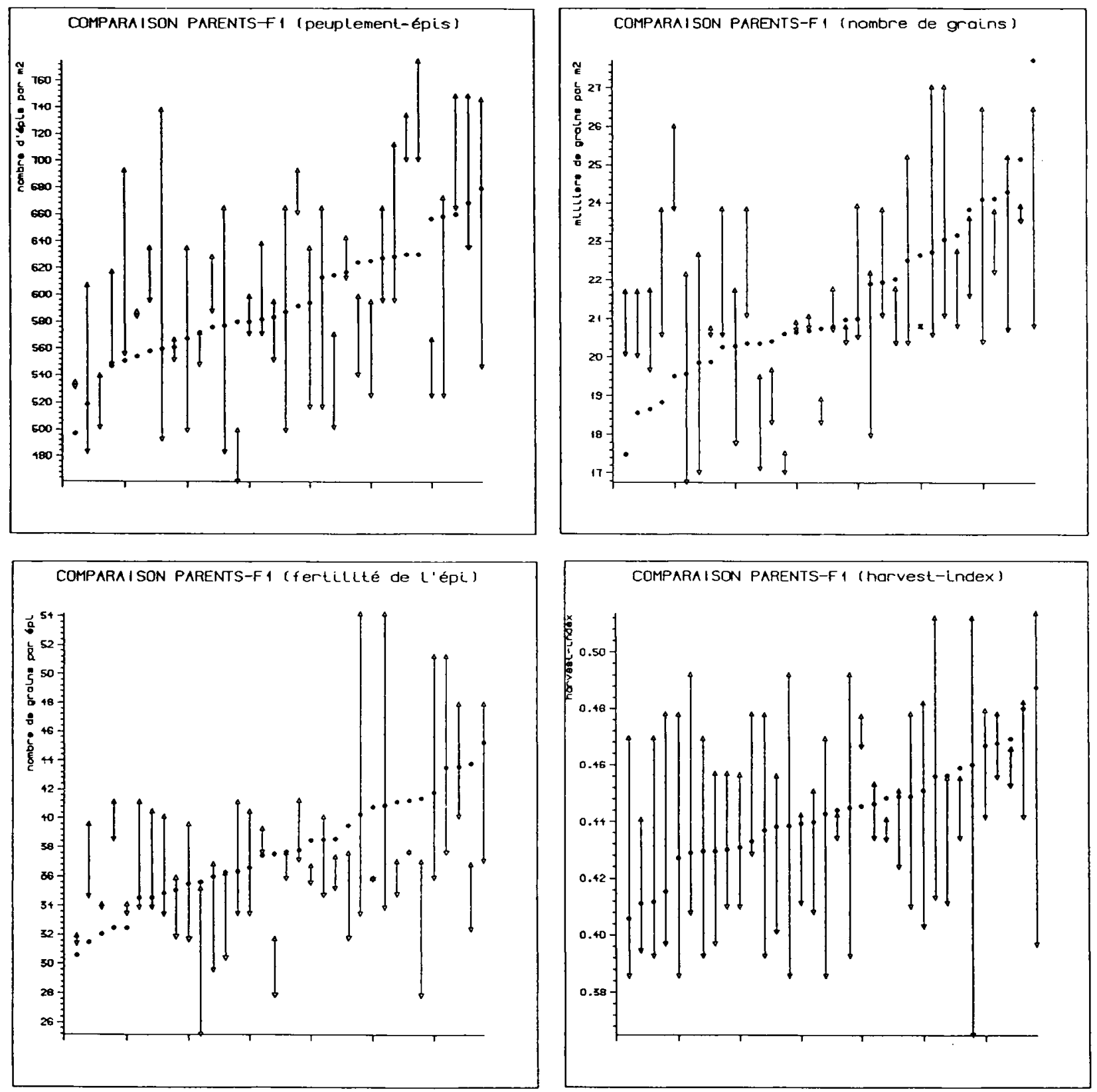

Fig 2. Valeurs observées pour 34 triplets constitués par 1 hybride $\left(^{*}\right)$, son parent inférieur $(\nabla)$, et son parent supérieur $(\Delta)$, pour 4 variables (HI, NE, NG et NGÉ) ne présentant pas globalement d'effet d'hétérosis. Les triplets hybride + parents sont disposés selon les valeurs croissantes des $F_{1}$.

d'une année à l'autre, excepté pour le harvestindex, la hauteur, et la précocité. Par ailleurs, le tableau $\mathrm{V}$ indique que l'instabilité des hybrides est du même ordre de grandeur que celle qui affecte leurs parents.

L'homéostasie du développement, souvent évoquée en faveur de l'utilisation de variétés hybrides dans le cas de différentes espèces autogames (Bingham, 1983; Lucken, 1986; LefortBuson, 1986; Rousset, 1986), n'apparaît donc pas dans notre étude. Bien que cela demande à être confirmé par des expérimentations portant sur un plus grand nombre de combinaisons hybrides, il semble donc que l'évaluation multilocale et pluriannuelle des $F_{1}$ reste nécessaire, ce qui rejoint les conclusions de Wienhues (1967), Nettevich (1968), Boland et Walcott (1985), Lucken (1986), Smith et al $(1987,1988)$. II faut cependant noter que ces réactivités au milieu, comparables pour les $F_{1}$ et les lignées, ont été obtenues seulement en conditions de culture intensive. Ceci ne préjuge donc pas d'une possible différence de réponse entre les hybrides et leurs parents, pour des conditions de culture plus ex- 


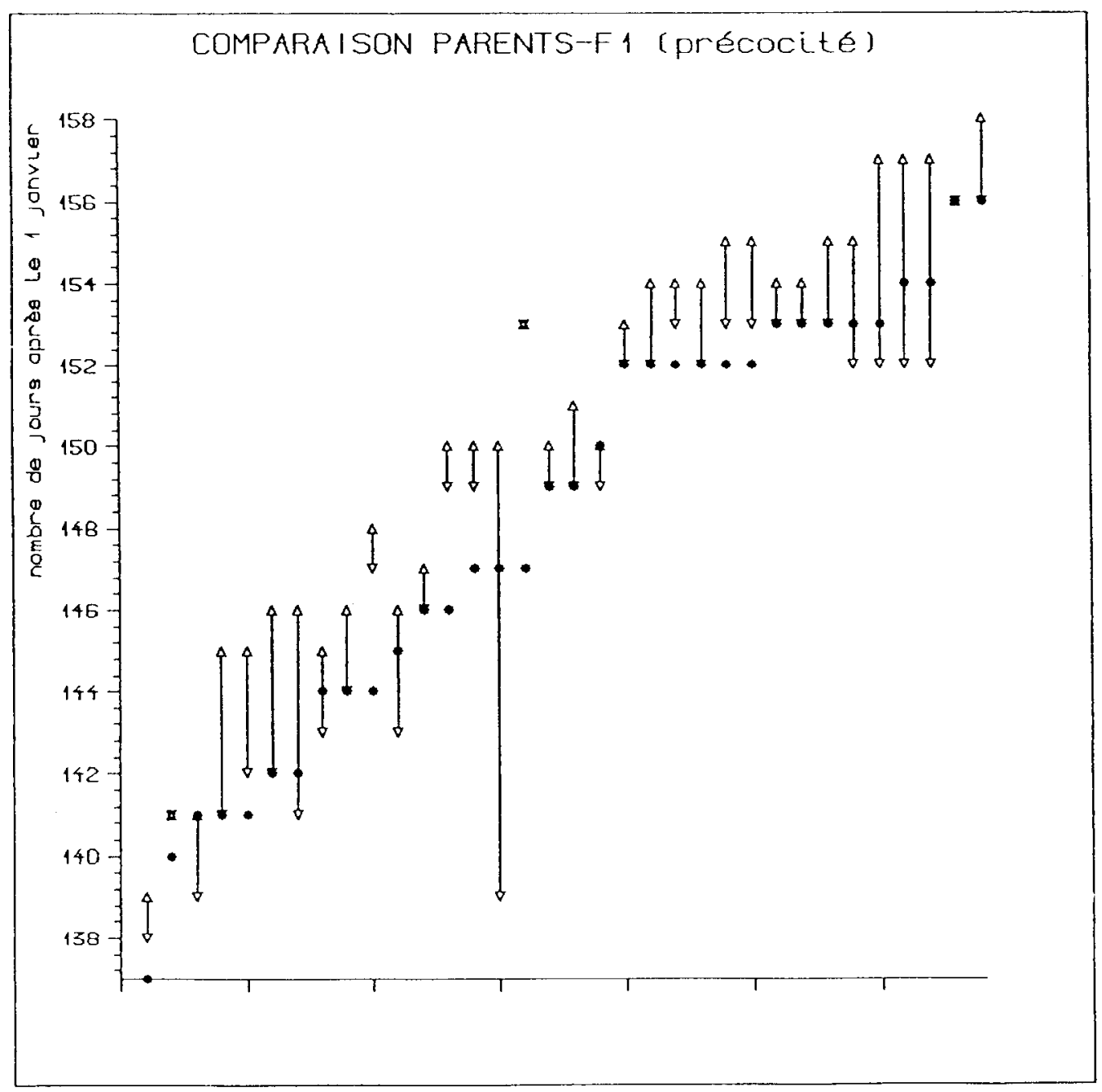

Fig 3. Précocité d'épiaison pour 34 triplets constitués par 1 hybride $\left.{ }^{*}\right)$, son parent inférieur $(\nabla)$, et son parent supérieur $(\Delta)$. Les triplets hybride + parents sont disposés selon les valeurs croissantes des $F_{1}$.

tensives. En effet, l'homéostasie théoriquement attendue chez l'hybride pourrait éventuellement permettre une réduction des traitements phytosanitaires, ou une diminution de la densité de semis (Triboi et Rousset, 1986).

\section{Lien entre valeur de l'hybride et valeur des parents}

On constate (tableau VI) une forte corrélation positive entre le rendement de l'hybride d'une part, et l'hétérosis parent supérieur (ou parent moyen) pour le rendement d'autre part. De même, il apparaît une corrélation négative entre l'hétérosis parent supérieur (ou parent moyen) pour le rendement d'une part, et le rendement du parent supérieur (ou du parent moyen) d'autre part : les hybrides ayant les parents de plus faible valeur propre sont donc également ceux qui présentent les niveaux d'hétérosis les plus élevés. Cela se traduit, au niveau de notre échantillon, par une indépendance entre le rendement de l'hybride d'une part, et le rendement du parent supérieur ou celui du parent moyen d'autre part (tab VI).

Ce dernier résultat ne correspond pas à celui de Morgan et al (1989), qui mettent en évidence une corrélation positive significative entre le rendement de l'hybride et celui du parent supérieur. Ceci n'est pas forcément contradictoire, compte tenu des différences de taille des échantillons étudiés : Morgan et al (1989) ont travaillé sur 430 hybrides, et la variabilité au sein des génotypes parentaux était donc certainement plus élevée dans leur cas que dans le nôtre.

Remarque : on peut noter qu'avec nos propres données, mais lorsqu'on ne corrige pas l'effet année, il apparaît également une relation étroite entre le rendement de l'hybride et celui du parent supérieur. En effet, la régression linéaire rendement $t_{\text {hybride }}=f($ rendement parent supérieur $)$ 
Tableau IV. Hétérosis par rapport au parent moyen et au parent supérieur, pour les 9 caractères étudiés et pour 3 hybrides observés pendant plusieurs années.

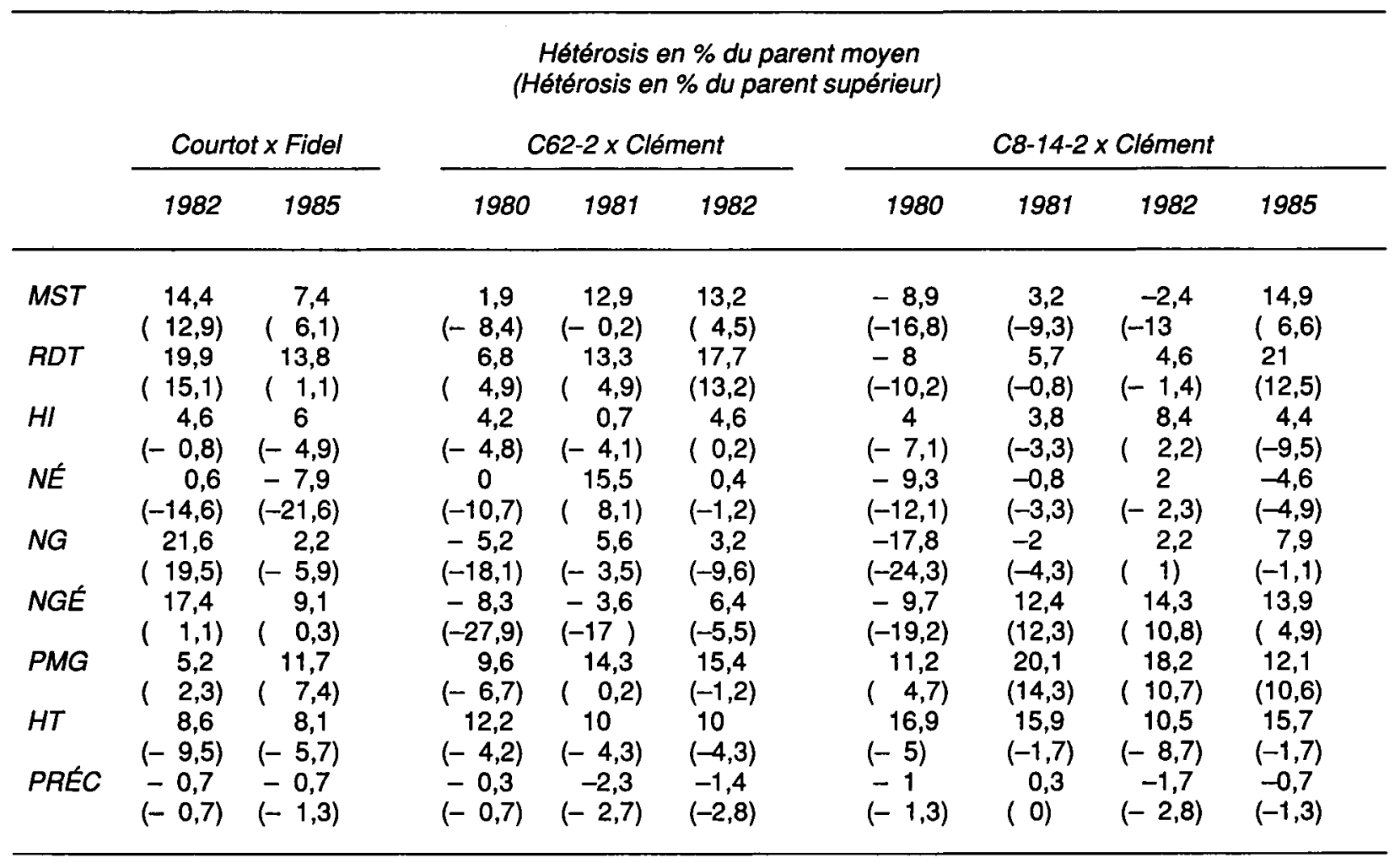

devient significative au seuil de $1 \%$, avec un coefficient de détermination $r^{2}=0,473$. Cependant, dans notre cas cette corrélation est essentiellement d'origine environnementale : elle est due à une augmentation de l'étendue du nuage de points sous l'action de l'effet année (les années les plus favorables à l'expression du rende- ment l'étant à la fois pour les hybrides et pour leurs parents). Par contre, dans le cas de Morgan et al (1989), l'augmentation de l'étendue du nuage de points est due à l'accroissement de la taille de l'échantillon, et la corrélation apparaît en raison de la plus grande variabilité des génotypes étudiés.

Tableau V. Écart-type inter annuel des moyennes intra-essais, pour les 9 caractères étudiés et pour 3 hybrides et leurs parents observés pendant plusieurs années.

\begin{tabular}{|c|c|c|c|c|c|c|c|c|c|}
\hline & \multicolumn{3}{|c|}{$\begin{array}{l}\text { Écart-type des moyennes } \\
\text { intra-essais sur } 2 \text { ans } \\
\text { (1982 et 1985) }\end{array}$} & \multicolumn{3}{|c|}{$\begin{array}{l}\text { Ecart-type des moyennes } \\
\text { intra-essais sur } 3 \text { ans } \\
(1980,1981 \text { et } 1982)\end{array}$} & \multicolumn{3}{|c|}{$\begin{array}{c}\text { Ecart-type des moyennes } \\
\text { intra-essais sur } 4 \text { ans } \\
(1980,1981,1982 \text { et 1985) }\end{array}$} \\
\hline & Courtot & $C \times F$ & Fidel & $62-2$ & $62-2 \times \mathrm{Cl}$ & Clément & $8-14-2$ & $8-14-2 \times \mathrm{Cl}$ & Clément \\
\hline MST & 33,1 & 25,7 & 20,3 & 15,5 & 17,8 & 26,8 & 21,3 & 29,2 & 22 \\
\hline$R D T$ & 21 & 14,6 & 8,6 & 8,4 & 5,8 & 8,7 & 14,1 & 15 & 7,1 \\
\hline$H I$ & 0,03 & 0,01 & 0,02 & 0,01 & 0,01 & 0,01 & 0,03 & 0,015 & 0,01 \\
\hline$N E$ & 161,6 & 97,2 & 84,1 & 17 & 47 & 75,6 & 62,5 & 53,8 & 85,9 \\
\hline$N G$ & 6614 & 3407 & 3230 & 1162 & 2288 & 2206 & 2805 & 4161 & 2089 \\
\hline$N G E ́$ & 2,9 & 0,3 & 4,1 & 4,1 & 6,7 & 6,8 & 2,2 & 7,7 & 5,8 \\
\hline$P M G$ & 6,5 & 5,2 & 4,9 & 2,2 & 2,6 & 4,3 & 3 & 4,1 & 4,7 \\
\hline$H T$ & 6,4 & 3,5 & 4,4 & 0 & 2,9 & 2,9 & 3,2 & 4,4 & 2,4 \\
\hline PRÉC & 5,7 & 6,4 & 5,1 & 6,1 & 6 & 4,5 & 5 & 5,3 & 4,3 \\
\hline
\end{tabular}


Tableau VI. Caractéristiques de différentes régressions linéaires mettant en évidence les liens existant entre le rendement de l'hybride, l'hétérosis pour le rendement, et le rendement des parents. "* = significatif au seuil de $1 \%$.

\begin{tabular}{|c|c|c|c|}
\hline $\begin{array}{l}\text { Variable } \\
\text { expliquée } \\
\text { (y) }\end{array}$ & $\begin{array}{l}\text { Variable } \\
\text { explicative } \\
(x)\end{array}$ & $\begin{array}{c}\text { Équation de la } \\
\text { droite de régression } \\
\text { (significativité) }\end{array}$ & $\begin{array}{c}\text { Coefficient de } \\
\text { détermination } \\
\text { (coeff de corrélation) }\end{array}$ \\
\hline $\begin{array}{l}\text { Rendement de } \\
\text { l'hybride }\end{array}$ & $\begin{array}{l}\text { Hétérosis par rapport } \\
\text { au parent supérieur }\end{array}$ & $\begin{array}{c}y=32,53+0,514 x \\
(* *)\end{array}$ & $\begin{array}{c}0,648 \\
(0,805)\end{array}$ \\
\hline $\begin{array}{l}\text { Rendement de } \\
\text { l'hybride }\end{array}$ & $\begin{array}{l}\text { Hétérosis par rapport } \\
\text { au parent moyen }\end{array}$ & $\begin{array}{c}y=29,165+0,522 x \\
\left(^{* \star}\right)\end{array}$ & $\begin{array}{c}0,750 \\
(0,866)\end{array}$ \\
\hline $\begin{array}{l}\text { Hétérosis par rapport } \\
\text { au parent supérieur }\end{array}$ & $\begin{array}{l}\text { Rendement du } \\
\text { parent supérieur }\end{array}$ & $\begin{array}{c}y=213,84-1,332 x \\
\left(^{\star \star}\right)\end{array}$ & $\begin{array}{c}0,407 \\
(-0,638)\end{array}$ \\
\hline $\begin{array}{l}\text { Hétérosis par rapport } \\
\text { au parent moyen }\end{array}$ & $\begin{array}{l}\text { Rendement du } \\
\text { parent moyen }\end{array}$ & $\begin{array}{c}y=248,68-1,772 x \\
\left(^{* *}\right)\end{array}$ & $\begin{array}{c}0,448 \\
(-0,669)\end{array}$ \\
\hline $\begin{array}{l}\text { Rendement } \\
\text { de l'hybride }\end{array}$ & $\begin{array}{l}\text { Rendement du } \\
\text { parent supérieur }\end{array}$ & $\begin{array}{c}y=92,07-0,079 x \\
\text { (NS) }\end{array}$ & $\begin{array}{c}0,003 \\
(-0,055)\end{array}$ \\
\hline $\begin{array}{l}\text { Rendement } \\
\text { de l'hybride }\end{array}$ & $\begin{array}{l}\text { Rendement du } \\
\text { parent moyen }\end{array}$ & $\begin{array}{c}y=112,28-0,337 x \\
\text { (NS) }\end{array}$ & $\begin{array}{r}0,045 \\
(-0,212)\end{array}$ \\
\hline
\end{tabular}

En définitive, Morgan et al (1989) montrent que les hybrides obtenus à partir de parents ayant une faible valeur propre sont moins bons que ceux produits avec des parents de bonne valeur propre. Alors que nos résultats indiquent que pour un échantillon parental assez homogène et de bonne valeur propre, il est difficile de prédire le rendement de la $F_{1}$ à partir de celui de ses parents.

\section{Élaboration du rendement de l'hybride}

Le rendement peut s'exprimer sous la forme (Triboi et Rousset, 1986) :

$R D T=N G \times(P M G / 1000)=(N E ́ \times N G E) \times$ (PMG/1 000)

où $R D T$ est exprimé en $\mathrm{g} / \mathrm{m}^{2}$.

En situation de compétition entre plantes (ce qui est le cas avec le niveau de densité retenu

Tableau VII. Caractéristiques de différentes régressions linéaires mettant en évidence les liens existant entre le rendement et ses composantes, pour les hybrides, les parents, et la population globale (hybrides + parents). ${ }^{* *}=$ significatif au seuil de $1 \% ;{ }^{* * *}=$ significatif au seuil de $1 \%$.

\begin{tabular}{|c|c|c|c|c|}
\hline \multirow{2}{*}{$\begin{array}{l}\text { Variable } \\
\text { expliquée } \\
\text { (y) }\end{array}$} & \multirow{2}{*}{$\begin{array}{l}\text { Variable } \\
\text { explicative } \\
\quad(x)\end{array}$} & \multicolumn{3}{|c|}{$\begin{array}{c}\text { Équation de la droite de régression } \\
\text { (significativité) } \\
\text { (coefficient de détermination) }\end{array}$} \\
\hline & & Pour les parents & Pour les hybrides & $\begin{array}{l}\text { Pour l'ensemble des géno- } \\
\text { types }\end{array}$ \\
\hline $\begin{array}{l}\text { Nombre de grains } \\
\text { par épi }\end{array}$ & $\begin{array}{l}\text { Nombre d'épis } \\
\text { par } \mathrm{m}^{2}\end{array}$ & $\begin{array}{c}y=60,3-0,04 x \\
\left({ }^{\star \star *}\right) \\
(0,344)\end{array}$ & $\begin{array}{c}y=61,2-0,039 x \\
(\stackrel{* *}{* *}) \\
(0,226)\end{array}$ & $\begin{array}{c}y=61,5-0,041 x \\
\left({ }^{* \star *}\right) \\
(0,320)\end{array}$ \\
\hline $\begin{array}{l}\text { Poids de } \\
1000 \text { grains }(g)\end{array}$ & $\begin{array}{l}\text { Nombre de grains } \\
\text { par } m^{2}\left(10^{3}\right)\end{array}$ & $\begin{array}{c}y=69,6-1,419 x \\
\left(\begin{array}{c}(* *) \\
(0,646)\end{array}\right.\end{array}$ & $\begin{array}{c}y=66,9-1,11 x \\
(* * *) \\
(0,512)\end{array}$ & $\begin{array}{c}y=68,3-1,274 x \\
\left(\begin{array}{c}* * \star \\
(0)\end{array}\right. \\
(0,470)\end{array}$ \\
\hline
\end{tabular}




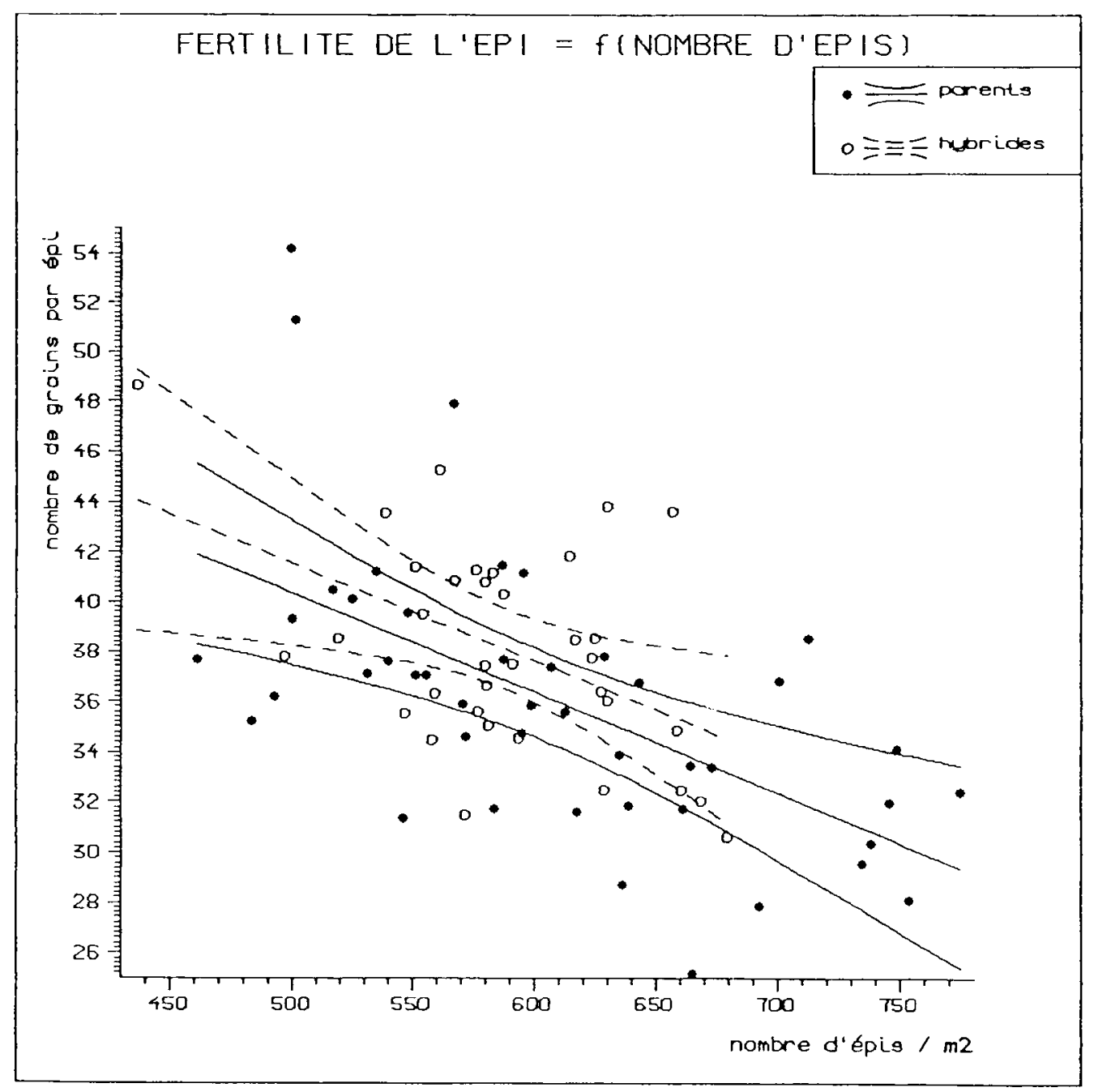

Flg 4. Régression linéaire du nombre de grains par épi sur le nombre d'épis par $\mathrm{m}^{2}$, pour les parents et les hybrides (les courbes encadrant les droites de régression délimitent la région de confiance à $95 \%$ de ces droites).

pour nos essais), et donc en présence de facteurs limitants, il y a des corrélations négatives entre $N G$ et $P M G$, ainsi qu'entre $N E ́$ et $N G E$, qui traduisent les compensations entre composantes qui interviennent au cours de l'élaboration du rendement (Triboi et al, 1985; Triboi, 1986).

Une régression linéaire, avec $N E ́$ comme variable explicative et $N G E$ comme variable expliquée, met en évidence une loi unique pour l'ensemble des génotypes (tableau VII et fig 4). En effet, un modèle de régression multiple basé sur l'existence de 2 équations différentes - l'une pour les hybrides, l'autre pour les parents - (Tomassone et al, 1983) ne permet pas une meilleure analyse de la relation $(F=0,857$ à 2 et $76 \mathrm{ddl}: P>0,05)$. Pour un même niveau de peuplement-épis, l'hybride ne produit donc pas plus de grains par $\mathrm{m}^{2}$ qu'une lignée, et la compensation entre $N G$ et $N G E ́$ joue de la même façon chez l'un et chez l'autre.
Par contre, une régression linéaire, faisant intervenir NG comme variable explicative et $P M G$ comme variable expliquée (tableau VII et fig 5), met en évidence une différence entre la population des hybrides et celle des parents. En effet, le modèle de régression multiple basé sur la distinction des 2 sous-populations permet un apport très significatif $(F=23,590$ à 2 et $76 \mathrm{ddl}: P<$ $0,01)$ par rapport au modèle de régression linéaire simple appliqué à l'ensemble des génotypes. L'observation de la figure 5 nous permet de conclure qu'à nombre de grains par $\mathrm{m}^{2}$ équivalent, un hybride remplit mieux ses grains qu'une lignée. Cependant, notre expérimentation ne permet pas de déterminer s'il s'agit d'un gain au niveau de la vitesse ou de la durée de remplissage. Par ailleurs, le parallélisme entre les 2 droites de régression indique que la compensation entre NG et $P M G$ est similaire dans les 2 cas. 


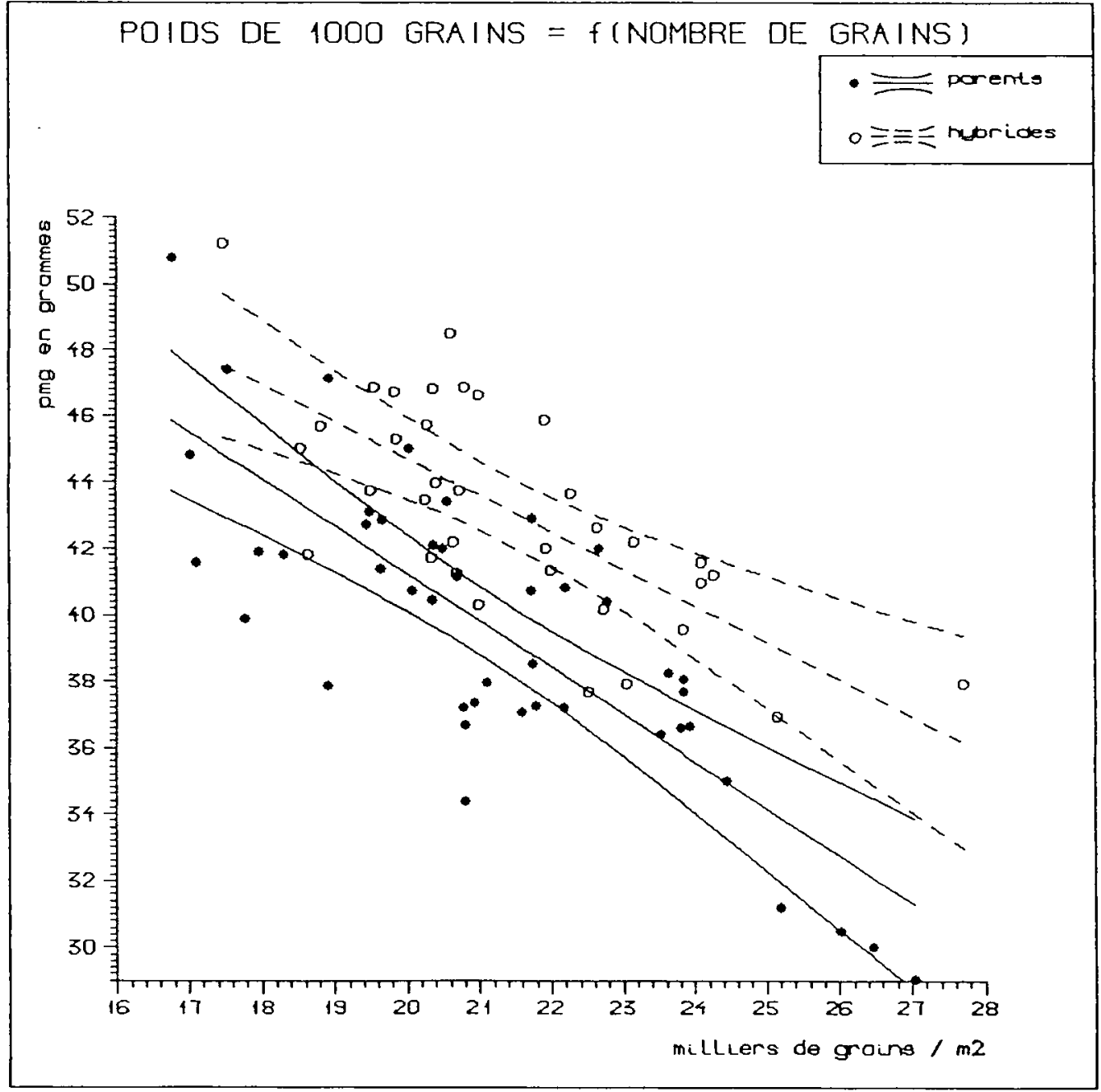

Fig 5. Régression linéaire du poids de 1000 grains sur le nombre de grains par $\mathrm{m}^{2}$, pour les parents et pour les hybrides (les courbes encadrant les droites de régression délimitent la région de confiance à $95 \%$ de ces droites).

On constate (tableaux VIII et IX), qu'il n'y a aucune corrélation entre le rendement de l'hybride et le poids de 1000 grains, non plus qu'entre ce rendement ou l'hétérosis sur le rendement, et l'hétérosis sur le poids de 1000 grains. Par contre, le rendement de la $F_{1}$ et l'hétérosis sur le rendement apparaissent fortement liés au nombre de grains par $\mathrm{m}^{2}$ produit par l'hybride, et

Tableau VIII. Coefficients de corrélation linéaire entre le rendement de l'hybride d'une part, et les valeurs et les niveaux d'hétérosis obtenus pour 7 caractères d'autre part.

Corrélations entre rendement de l'hybride et

\begin{tabular}{ccc}
\hline $\begin{array}{c}\text { La valeur de } \\
\text { I'hybride }\end{array}$ & $\begin{array}{c}\text { La valeur de I'hétérosis par } \\
\text { rapport au parent moyen }\end{array}$ & $\begin{array}{c}\text { La valeur de l'hétérosis par } \\
\text { rapport au parent supérieur }\end{array}$ \\
0,778 & 0,787 & 0,710 \\
1,000 & 0,866 & 0,805 \\
0,190 & 0,402 & 0,357 \\
0,719 & 0,743 & 0,558 \\
0,496 & 0,312 & 0,205 \\
$-0,111$ & 0,112 & 0,041 \\
0,299 & 0,356 & 0,302 \\
\hline
\end{tabular}


Tableau IX. Coefficients de corrélation linéaire entre la valeur de l'hétérosis pour le rendement d'une part, et les valeurs d'hétérosis observées pour 7 caractères d'autre part.

Corrélations entre l'hétérosis sur le rendement et

$\begin{array}{lrr}M S T & 0,921 & 0,789 \\ R D T & 1,000 & 1,000 \\ N E & 0,479 & 0,454 \\ N G & 0,872 & 0,704 \\ N G E & 0,382 & 0,219 \\ P M G & 0,008 & -0,066 \\ H I & 0,393 & 0,386\end{array}$

à l'hétérosis pour ce nombre de grains. Cela signifie que, par rapport à un phénomène de meilleur remplissage du grain qui est commun à l'ensemble des $F_{1}$, les combinaisons hybrides vraiment performantes sont celles qui présentent, en plus, un hétérosis pour le nombre de grains par $\mathrm{m}^{2}$.

Malheureusement, ce nombre de grains produit par $\mathrm{m}^{2}$ semble difficile à employer pour la sélection des blés hybrides car, d'une part, il ne peut pas être déterminé de manière précoce au cours du cycle végétatif, d'autre part il ne peut pas être mesuré de manière valable sur un dispositif expérimental restreint. Cependant, les coefficients de corrélation entre le nombre de grains produit par l'hybride d'une part, et le nombre de grains produit par le parent moyen ou le parent supérieur d'autre part, valent respectivement 0,498 et 0,502 . II paraît donc possible d'utiliser l'information éventuellement disponible pour ce caractère au niveau des parents, pour une prédiction de la valeur de l'hybride.

Enfin, on observe (tableau VIII) une corrélation entre le rendement de l'hybride et la quantité de matière sèche produite. Cette relation est d'ailleurs beaucoup moins forte chez les parents (le coefficient de corrélation vaut seulement 0,488 ), sans doute en raison de la présence de nombreuses lignées demi-naines. On observe surtout (tableau $(X)$, une corrélation étroite entre l'hétérosis sur le rendement et l'hétérosis sur la matière sèche aérienne. La voie hybride pourrait donc être un moyen de sortir du schéma en vigueur depuis plusieurs décennies dans l'amélioration du blé, et qui consiste en une augmentation du harvest-index (celui-ci était voisin de 30\% pour les variétés anciennes, et approche maintenant $50 \%$ pour un blé moderne possédant au moins 1 des gènes du nanisme) sans accroissement de la quantité de biomasse aérienne produite (Austin et al, 1980).

\section{CONCLUSION}

La manifestation de la vigueur hybride au niveau du rendement apparaît d'une manière nette dans notre étude, et la valeur que nous obtenons pour I'hétérosis par rapport au parent supérieur $(3,1 \%$ pour 34 hybrides) est comparable aux valeurs rapportées pour des essais réalisés dans des conditions expérimentales proches des nôtres : 4,3\% pour 10 hybrides (Borghi et al, 1988), 7,3\% pour 17 hybrides (Brears et al, 1988), 5,9\% et $3,6 \%$ pour respectivement 61 et 430 hybrides (Morgan et al, 1989).

Les semences $F_{1}$ sont considérablement plus chères que les semences des lignées pures. En effet, au coût du traitement gamétocide s'ajoute une diminution de la production à l'hectare, du fait d'éventuels problèmes de stérilité-femelle partielle sur les plantes traitées, de la moindre efficacité et des aléas de l'allopollinisation et, dans une moindre mesure, de la non-récolte des bandes mâles. Les valeurs d'hétérosis rapportées ci-dessus peuvent alors sembler faibles au regard des niveaux que le calcul économique fait apparaître comme nécessaires pour compenser le surcoût : $11 \%$ pour Rousset (1986), 13\% pour Brears et al (1988).

Cependant la variabilité au niveau de ces valeurs d'hétérosis est importante. L'écart type de I'hétérosis parent supérieur pour le rendement est de 6,9 dans le cas des 61 hybrides de l'étude de Morgan et al, et vaut 8,4 dans notre étude, pour laquelle l'échantillon parental était pourtant 
assez homogène car constitué de lignées toutes de bonne valeur propre. II semble donc possible de faire une sélection valable des parents sur le critère de l'aptitude à la combinaison, et d'arriver ainsi à des niveaux d'hétérosis plus proches des seuils économiques acceptables.

D'autre part, il ne faut pas oublier que ces seuils économiques ont été déterminés en considérant des conduites de culture uniformes pour les lignées et les hybrides. Or la compétitivité des $F_{1}$ par rapport aux lignées pourrait être accrue $s^{\prime} i l$ s'avérait que leur culture requiert des doses moins fortes de certains intrants. II serait donc intéressant de réaliser des expérimentations pour voir s'il y a une réponse particulière des hybrides aux faibles densités de semis, ou à des traitements phytosanitaires réduits.

Enfin, il faut noter qu'aucune des combinaisons hybrides que nous avons étudiées n'était demi-naine : si l'augmentation du harvest-index que l'on a enregistrée au niveau des lignées pures après introduction des gènes de nanisme, se retrouve au niveau des hybrides, et s'associe à l'accroissement de biomasse dû à l'hétérosis, il y a là un gisement de productivité qui pourrait rendre les blés hybrides commercialement attrayants.

\section{RÉFÉRENCES}

Akemine H, Kumagai K (1966) Heterosis in wheat hybrids. Seiken Zihô 18, 64-75

Austin RB, Bingham J, Blackwell RD, Evans LT, Ford MA, Morgan CL, Taylor M (1980) Genetic improvements in winter wheat yields since 1900 and associated physiological changes. J Agric Sci Camb 94, 675-689

Bachacou J, Masson JP, Millier C (1981) Manuel de la programmathèque statistique AMANCE. INRA, 516 pp

Bingham J (1983) Genetic constraints on progress in wheat breeding. In: The yields of cereals, Proc Int Seminar, Cambridge and NAC Stamleigh, 5-11

Bitzer MJ, Patterson FL, Nyquist WE (1982) Hybrid vigor and combining ability in a high-low yielding, eight-parent diallel cross of soft red winter wheat. Crop Sci 22, 1 126-1 129

Boland OW, Walcott JJ (1985) Levels of heterosis for yield and quality in an $F_{1}$ hybrid wheat. Aust $J$ Agric Res 36, 545-552

Borghi B, Perenzin M, Nash RJ (1988) Agronomic and qualitative characteristics of ten bread wheat hy brids produced using a chemical hybridizing agent. Euphytica 39, 185-194

Borojevic $S$ (1966) Combining ability in wheat crosses. Hereditas, suppl vol 2, 102-118
Brears T, Hydon AG, Bingham J (1988) An assessment of the feasibility of producing $F_{1}$ and $F_{2}$ hy brids for the UK. Proc 7th Int Wheat Genet Symp, $1057-1062$

Briggle LW, Daum RJ, Stevens H (1964) Expression of heterosis in two wheat crosses. Crop Sci 4, 220223

Briggle LW, Petersen HD, Hayes RM (1967) Performance of a winter wheat hybrid, $F_{2}, F_{3}$, and parent varieties at five population levels. Crop Sci 7, 485490

Edwards I (1987) Comparison of hybrid and varietal wheat breeding. In: Hybrid and conventional variety selection considerations, Proc 2nd Nat Wheat Res Conf, Kansas City, February 1987, 10 pp

Glenn DE (1987) Seed wheat production: varieties and hybrids. In: Hybrid and conventional variety selection considerations. Proc 2nd Nat Wheat Res Conf, Kansas City, February 1987, 10 pp

Goujon C, Paquet J (1968) Contribution à l'étude de la vigueur hybride chez le blé tendre : essais de rendements en semis d'automne réalisés en 196566 et 1966-67. Ann Amelior Plant 18, 223-235

Ingold M (1974) La longueur de la paille dans les hybrides $\mathrm{F}_{1}$ du blé (Triticum aestivum $\mathrm{L}$ ). Rech Agron Suisse 13, 235-243

Jost $M$, Hayward CF (1980) $F_{1}$ hybrids versus 32 selected $F_{7}$ lines performance of common winter wheat (Triticum aestivum ssp vulgare). Theor Appl Genet 57, 177-180

Keydel F (1973) Untersuchung über die Leistungseigenschaften von $F_{1}$-Hybriden von Winterweizen. $Z$ Pflanzenzuecht 69, 239-255

Lefort-Buson M (1986) Hétérosis chez le colza oléagineux (Brassica napus $L$ ) : analyse génétique et prédiction. Thèse d'État de l'université Paris-Sud Orsay, $228 \mathrm{pp}$

Lucken KA (1986) The breeding and production of hybrid wheat. In: Genetic improvement in yield of wheat, Proc CSSA Symp CSSA Spec Publ, 87-107

Morgan CL, Austin RB, Ford MA, Bingham J, Angus WJ, Chowdhury $S$ (1989) An evaluation of $F_{f}$ hybrid winter wheat genotypes produced using a chemical hybridizing agent. J Agric Sci Camb 112, 143-149

Nettevich ED (1968) The problem of utilizing heterosis of wheat (Triticum aestivum L). Euphytica 17, 5462

Planchon C, Diehl R (1967) Quelques aspects du phénomène d'hétérosis chez le blé tendre (Triticum aestivum L). CR Ac Sci 264, 2 011-2 014

Rousset M (1984) Les gamétocides, qu'est-ce que c'est? Bulletin Semences de la FNAMS 88, 5-8

Rousset M (1986) Les blés hybrides sortent du laboratoire. Recherche 17, 86-89

Sharma JC, Ahmad Z (1978) Economic heterosis in relation to heterotic parameter in spring wheat. Indian J Genet \& Plant Breed 38, 361-371 
Smith EL, Sharma RC, Morgan GH, Carver BF, Merrit RG (1987) Results of 1986 wheat variety-hybrid performance test. Research report P-889, Oklahoma Agric Experiment Station, $22 \mathrm{pp}$

Smith EL, Morgan GH, Sharma RC, Tahara M (1988) Results of 1987 wheat variety-hybrid performance test. Research report p-901, Oklahoma Agric Experiment Station, $24 \mathrm{p}$

Tomassone R, Lesquoy E, Millier C (1983) La régression : nouveaux regards sur une ancienne méthode statistique. INRA et Masson, Paris, $184 \mathrm{p}$

Triboi E (1986) Étude du potentiel génétique de production du blé tendre à travers la structure et le fonctionnement du peuplement. In : Rapport intermédiaire de l'ATP Ecophysiologie du Blé, INRA, 93103

Triboi E, Rousset M (1986) L'élaboration du rendement chez les blés hybrides en comparaison avec les parents. In: Rapport intermédiaire de l'ATP Ecophysiologie du Blé, INRA, 313-332

Triboi E, Blanchon J, Magne J (1985) Déterminisme du poids moyen du grain chez le blé. Effet sur la variation du rendement. CR Acad Agric 71, 871-886

Wienhues $F$ (1967) Long-term yield analyses about heterosis in wheat and barley : variability of heterosis, fixation of heterosis. Communication au congrès Eucarpia, Wageningen, octobre 1967 